\title{
Investigations of the high-pressure, high-temperature behaviour of Au using laser-driven dynamic compression
}

\author{
A.L. Coleman, R.F. Smith, T. Lockard, D.C. Swift, J.M. McNaney \\ Lawrence Livermore National Laboratory, Livermore, California 94500, USA \\ coleman55@llnl.gov
}

Au has long been regarded as an important calibration standard in the high-pressure diffraction community, especially for experiments involving diamond anvil cells. The face centred cubic phase of $\mathrm{Au}$ is believe to be stable for hundreds of GPa at room temperature $[1,2]$. Recent dynamic-compression work has shown that the high-pressure behaviour of $\mathrm{Au}$ is not as simple at higher temperatures, and under laser-driven shock-compression, Au was found to transform, on-Hugoniot, from its ambient face centred cubic phase to a body centred cubic phase at $223 \mathrm{GPa}$ before melting around $320 \mathrm{GPa}$ [3].

As well as being used as a calibration standard, Au is also a commonly used material in target packages for laser-driven, dynamiccompression experiments. For experiments that explore the behaviour of various materials at the highest pressures and temperatures achievable (such as the experiments conducted at the National Ignition Facility or at the Omega laser facility) a layer of Au may be placed before the material of interest to act as a shield to prevent $\mathrm{x}$-ray heating of the material of interest before the compression wave has reached the sample. For many of these experiments, the compression wave is not necessarily a shock wave, but the target may instead be ramp-compressed meaning that the compression state does not lie on the Hugoniot.

Given the frequent use of $\mathrm{Au}$ in diffraction experiments at extreme conditions, it is important that its high-pressure, high-temperature behaviour is well constrained off-Hugoniot so that we may correctly identify its contribution to diffraction data collected in this regime. To this end, a series of shock and ramp compression experiments have been conducted across various laser-compression platforms to explore the extent of the high-pressure bcc phase of Au. These experiments involve the compression of Au to previously unexplored pressures and temperatures, utilizing diffraction and velocity interferometry as the primary diagnostics. This talk presents a discussion of these results and reconciles this new, unpublished data with existing findings within the field.

This work was performed under the auspices of the U.S. Department of Energy by Lawrence Livermore National Laboratory under Contract DE-AC52-07NA27344.

[1] Dubrovinsky, L., Dubrovinskaia, N., et al. (2007). Phys. Rev. Lett. 98, 045503

[2] Boettger, J.C. (2003) Phys. Rev. B. 67, 174107

[3] Briggs, R.J., et al., (2019) Phys. Rev. Lett. 123, 045701

Keywords: Dynamic-compression; High-pressure; Ultrafast-diffraction 